\title{
Gut microbiota as the key controllers of "healthy" aging of elderly people
}

Emeline Ragonnaud and Arya Biragyn ${ }^{*}$ (i)

\begin{abstract}
Extrinsic factors, such as lifestyle and diet, are shown to be essential in the control of human healthy aging, and thus, longevity. They do so by targeting at least in part the gut microbiome, a collection of commensal microorganisms (microbiota), which colonize the intestinal tract starting after birth, and is established by the age of three. The composition and abundance of individual microbiota appears to continue to change until adulthood, presumably reflecting lifestyle and geographic, racial, and individual differences. Although most of these changes appear to be harmless, a major shift in their composition in the gut (dysbiosis) can trigger harmful local and systemic inflammation. Recent reports indicate that dysbiosis is increased in aging and that the gut microbiota of elderly people is enriched in pro-inflammatory commensals at the expense of beneficial microbes. The clinical consequence of this change remains confusing due to contradictory reports and a high degree of variability of human microbiota and methodologies used. Here, we present the authors' thoughts that underscore dysbiosis as a primary cause of aging-associated morbidities, and thus, premature death of elderly people. We provide evidence that the dysbiosis triggers a chain of pathological and inflammatory events. Examples include alteration of levels of microbiota-affected metabolites, impaired function and integrity of the gastrointestinal tract, and increased gut leakiness. All of these enhance systemic inflammation, which when associated with aging is termed inflammaging, and result in consequent aging-associated pathologies.
\end{abstract}

Keywords: Commensals, IgA, B cells, Aging

\section{Introduction}

Today, modern humans live markedly longer than their predecessors in the early twentieth century, due to the achievements of modern medicine and lifestyle improvement. The importance of external factors in delaying intrinsic causes of aging-associated pathologies and diseases, i.e., in uncoupling chronological age from physical decline, has been recognized since ancient times. The first prescription to reduce the problems associated with aging, such as dizziness, eye inflammation and ear pain, using a diet of gruel, raw honey, vegetable and fowl is recorded in the book Hygiene, written by the Greek physician Galen in

\footnotetext{
*Correspondence: biragyna@mail.nih.gov
Immunoregulation Section, Laboratory of Immunology and Molecular

* Correspondence: biragyna@mail.nih.gov
Immunoregulation Section, Laboratory of Immunology and Molecular Biology, National Institute on Aging, 251 Bayview Blvd, Suite 100, Baltimore, MD 21224, USA into bioactive short chain fatty acids (SCFAs), generates vitamins and nutrients, maintains the intestinal integrity,
}

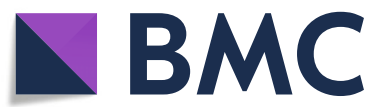

(c) The Author(s). 2021 Open Access This article is licensed under a Creative Commons Attribution 4.0 International License, which permits use, sharing, adaptation, distribution and reproduction in any medium or format, as long as you give appropriate credit to the original author(s) and the source, provide a link to the Creative Commons licence, and indicate if changes were made. The images or other third party material in this article are included in the article's Creative Commons licence, unless indicated otherwise in a credit line to the material. If material is not included in the article's Creative Commons licence and your intended use is not permitted by statutory regulation or exceeds the permitted use, you will need to obtain permission directly from the copyright holder. To view a copy of this licence, visit http://creativecommons.org/licenses/by/4.0/ The Creative Commons Public Domain Dedication waiver (http://creativecommons.org/publicdomain/zero/1.0/) applies to the data made available in this article, unless otherwise stated in a credit line to the data.

$175 \mathrm{AD}$ [1]. Then over century ago, Elie Metchnikoff postulated that senility is caused by "putrefactive bacterial autotoxins" leaked from the colon, and advocated a diet of fermented milk and a "simple" lifestyle to neutralize these autotoxins [2], for the first time emphasizing the importance of gut microbiota in human health and aging. The human gut microbiota is a commensal microbial "superorganism" that consists of trillions of Bacteria, Archaea, Eukarya and viruses, where four bacterial phyla (Firmicutes, Bacteroides, Proteobacteria and Actinobacteria) account for about $98 \%$ of the microorganisms. This superorganism coevolved with the host to provide numerous essential and mutually beneficial functions. It supports digestion and absorption of food, metabolizes fibers 
regulates host immunity and directly and indirectly protects from pathogens [3]. The gut microbiota is considered to be a master regulator of immune homeostasis [4], as its absence in germ-free (GF) mice impairs development and maturation of the immune system, while its presence in the gut induces IL-10 and TGF $\beta$-producing Tregs, immunoglobulin A (IgA)-secreting B cells, Th17 cells and type-2 lymphoid innate cells (ILC2) [5-9]. Oral supplementation of mice with 11 commensal strains of bacteria isolated from healthy human feces enhances the host resistance to Listeria monocytogenes infection and improves the therapeutic efficacy of immune checkpoint inhibitors in tumor-bearing mice through upregulation of IFNYexpressing $\mathrm{CD}^{+} \mathrm{T}$ cells in the intestine in a $\mathrm{CD} 103^{+}$ dendritic cell and MHC-I molecule-dependent manner [10]. The microbiota also supports maintenance of the intestinal mucus layer and induces production of various factors and secretory IgA from B cells to promote its own growth and to suppress pathogens.

Understanding the role of gut microbiota in human health is hampered by a high degree of variability.
Microbial composition differs depending on the conditions within the gastrointestinal tract, such as the high acidity of the stomach and the small intestine and the slightly acidic to neutral $\mathrm{pH}$ of the colon. Recent reports from sequencing human fecal microbiota revealed that composition of the gastro-intestinal microbes is affected by human inter-individual, racial, geographic and lifestyle differences. Furthermore, the abundance of its member composition changes depending on the physical state of the host. In people with morbid obesity, microbial composition in the gut shifts from providing benefit to causing harmful inflammation through at least in part impairing the intestinal epithelial integrity. Similar microbiota change has been proposed to occur also in aging and is thought to be a cause of various pathologies and diseases, such as frailty, neurodegeneration, insulin resistance and type- 2 diabetes (T2D), cancer, cardiovascular disease and Alzheimer's disease. Despite an explosion of reports that link gut microbiota to health in aging, the field remains poorly understood and appears to be confusing. Here, we briefly summarize findings from others and also from the authors'

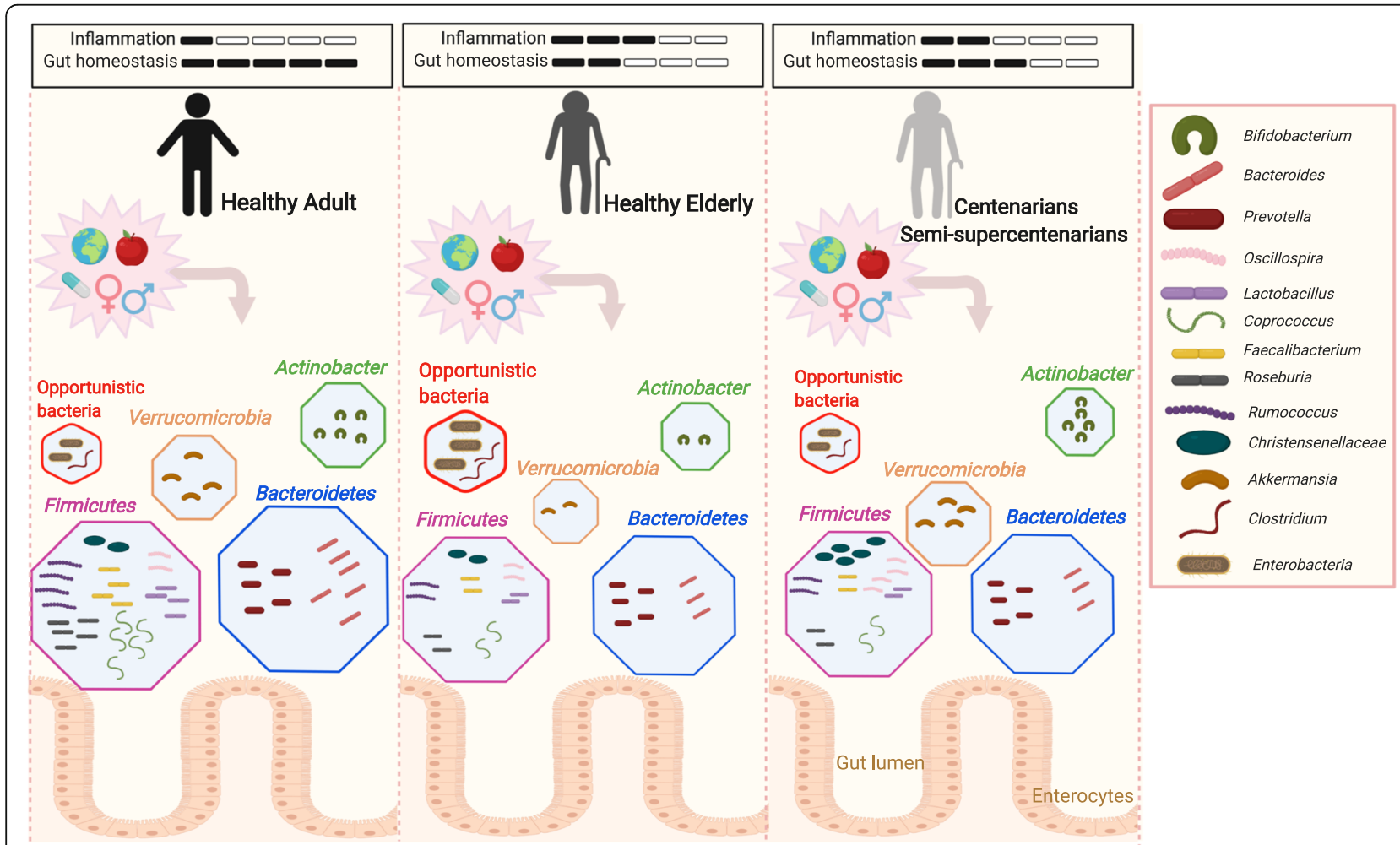

Fig. 1 The composition of gut microbiota determines inflammation and possibly lifespan of elderly people. The lumen and particularly the mucin layer of the intestine of young adults are colonized by a diverse population of commensal microbes that co-exist with the host in a symbiotic relationship. Members of Verrucomicrobia phylum, particularly Akkermansia muciniphila, support gut barrier integrity and thus prevent leakage and subsequent induction of inflammation. In elderly people, the composition of the gut commensals is changed and microbial diversity is reduced due to accumulation of potentially pro-inflammatory commensals and decrease of beneficial microbes, such as members of Verrucomicrobia. It therefore leads to gut leakiness and consequent systemic inflammation that facilitates aging-associated morbidities and premature death. Although the microbiota of centenarians changes, its diversity and beneficial commensals are retained, thereby controlling overt inflammation and supporting healthy aging 


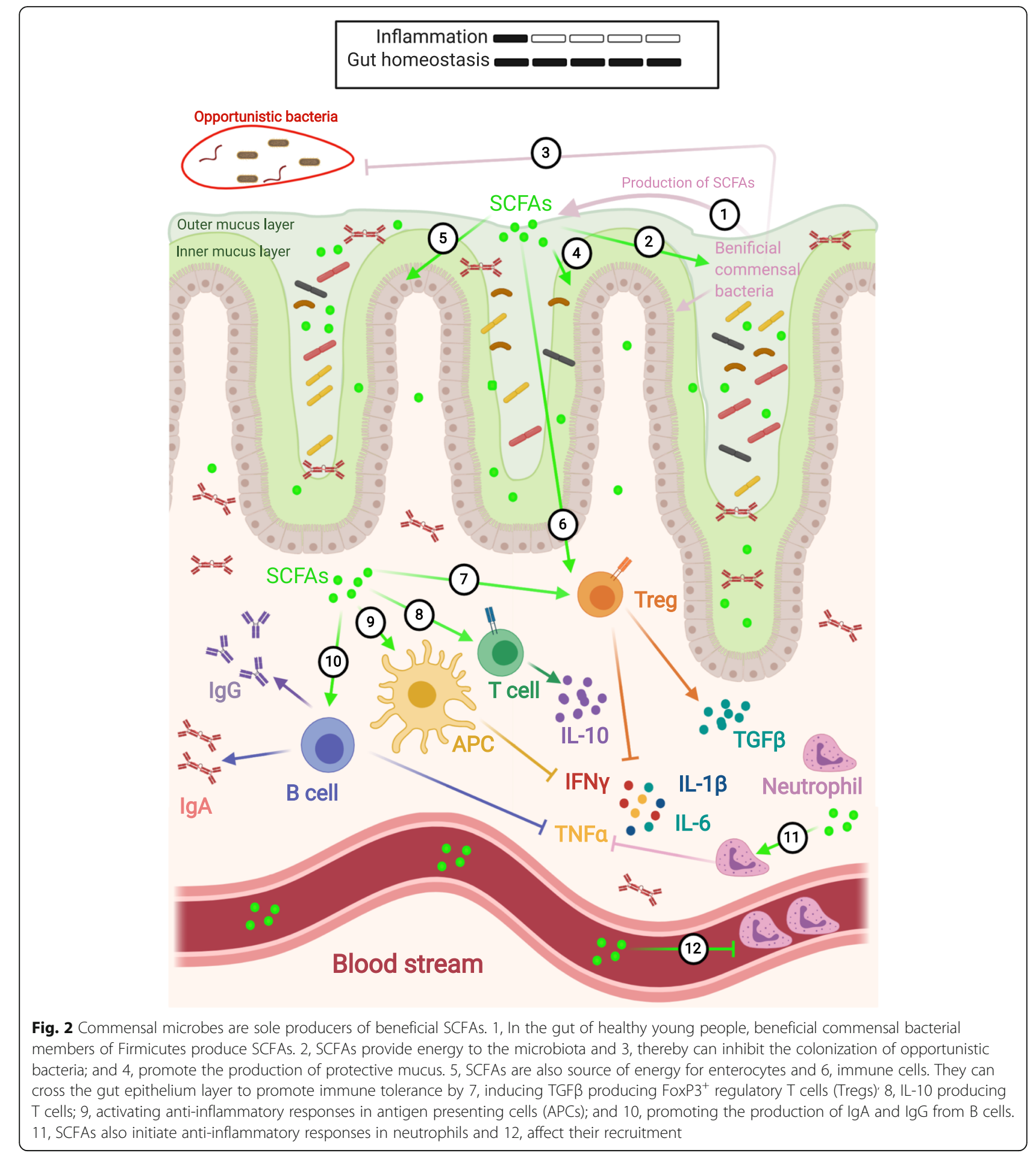

group to emphasize the importance of gut microbiota in the healthy aging of humans. Although dysbiosis and consequent inflammation in aging is assumed to be caused by western style diet (see review [11]), we discuss recent results of microbiota sequencing of elderly people from Italy and China, which suggest that the microbiota change could also be intrinsic to aging process. We propose that the change in gut microbiota is a primary cause of agingassociated pathologies and consequent premature death of elderly people. The most compelling evidence was revealed in studies of genetically homogeneous rodents, such as mice aged in the same environment. It showed that natural 


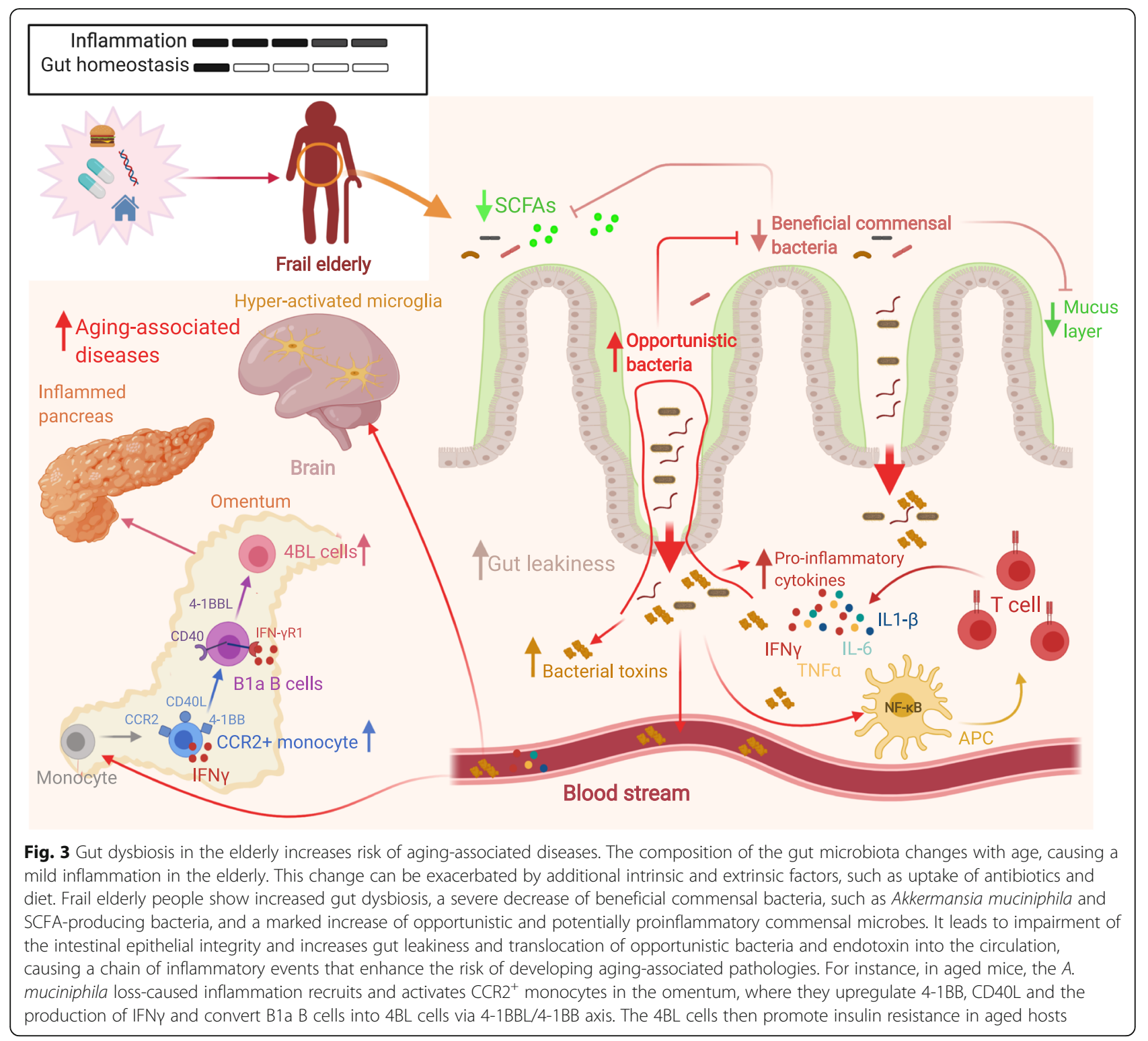

aging decreases the microbiota diversity and aged mice have diminished bacterial biosynthesis of cobalamin (B12) and biotin (B7), and SOS genes associated with DNA repair and enhanced creatine degradation, which is associated with muscle wasting [12-14]. Cross-sectional analyses of human fecal microbiota suggest that the fecal microbial population is also altered in elderly people, as in aged mice. The biological and, possibly, aging phenotype in humans appears to depend on four subpopulations (modules) of the "core" fecal microbiota [15]. Although it is difficult to link any given microbe to a clinical outcome, as it may require a cooperative action of multiple microbial species, we also discuss recent findings that underscore the importance of Akkermansia muciniphila in healthy aging. A. muciniphila is a bacterium that degrades mucin and provides energy to other beneficial microbes, including SCFA-producing bacteria. It also protects intestinal epithelial integrity via activation of epithelial cells and production of mucus, thus supporting housing of beneficial commensals. Its decrease in the gut of aged mice and, possibly, elderly people leads to gut leakiness and consequent induction of a low-level of systemic inflammation in aging (termed "inflammaging"). This presumably explains why increased levels of proinflammatory cytokines in the circulation are associated with an overall loss of fitness and poor health in the elderly $[16,17]$. However, the question remains unresolved whether the loss of Akkermansia causes or is caused by the decrease of microbiota diversity, which also occurs in aging and associates with frailty in elderly people [18]. The gut dysbiosis and the loss of beneficial 
commensals appear to facilitate premature death of elderly people, as fecal microbiota diversity and abundance of $A$. muciniphila are increased in human centenarians [19].

\section{Does the gut microbiota change with age?}

The gut microbiome is an endogenous ecosystem populated with Bacteria, Archaea, Eukarya and viruses, where four bacterial phyla of Firmicutes, Bacteroides, Proteobacteria and Actinobacteria account for $98 \%$ of microorganisms. It coevolved as a symbiotic superorganism with the host to regulate the normal functions of the gut, such as food digestion and absorption of nutrients, and to provide essential vitamins, nutrients, and polyamines. The microbiota degrades undigestible fibers and is thereby exclusively responsible for production of short-chain fatty acids (SCFAs) [20]. SCFAs are involved in multiple processes, such as providing an energy source for microbes and colonocytes, controlling microbial functions, combating pathogens, protecting and maintaining intestinal integrity, and regulating immune cells [3], including differentiation of $\mathrm{CD} 4^{+} \mathrm{T}$ cells and activation of $\mathrm{CD}^{+} \mathrm{T}$ cells [21-24]. As such, the absence of microbiota, e.g. SCFA-generating bacteria, in gnotobiotic (germ-free, GF) mice impairs nutrient absorption, dysregulates intestinal morphology, reduces differentiation and maturation of intestinal immune cells such as intraepithelial lymphocytes, Th17 cells, and regulatory $\mathrm{T}$ cells (Tregs). This causes shifts in immune responses towards Th2-type cytokines and impairs production of antimicrobial peptides and IgA. The gut microbiota is considered to be a master regulator of immune homeostasis [4], as it induces IL-10 and TGF $\beta$ producing regulatory $\mathrm{T}$ cells (Tregs), Th17 cells, ILC2 and IgA-secreting B cells [5-9]. For instance, members of Bacteroides fragilis and Clostridium strains induce $\mathrm{FoxP}^{+}$Treg differentiation and production of IL-10 and TGF $\beta$, resulting in the inhibition of inflammation. The intestinal segmented filamentous bacteria (SFB), Citrobacter rodentium and fungi like Candida albicans induce Th17 cell differentiation and recruitment of neutrophils and other immune cells in the lamina propria (LP), facilitating pathogen clearance.

The microbial colonization of the GI tract starts after the birth of infants by acquiring a few microbial types dominated by the Bifidobacterium genus [25]. The Bifidobacterium genus dominance declines after the first year of infancy as colonization with other microbes and their diversity increase [25]. The infant microbiota is unstable, and its microbial colonization process is influenced by the mode of delivery and nursing, medications, genetic background [26-33], age, and geographic/cultural traditions [25]. Breast-fed infants promote Bifidobacterium-dominated commensals with limited diversity primarily due to human milk oligosaccharides (HMOs), which Bifidobacterium species utilize and convert into lactate and short chain fatty acid acetate. In the absence of HMOs, the gut of formula-fed babies is colonized by a diverse population of microbes. The unstable state of the microbiota in infants presumably explains why the gut microbiota of US infants differs from that of non-US ones, as it is dominated with Prevotella genus including 28 Operational Taxonomic Units (OTU) [25]. This genus of mucin-degraders is also overrepresented in the fecal microbiota of children from Burkina Faso compared with children living in Italy [34]. The gut microbiota stabilizes when children start eating "solid food" at around 3 years of age and becomes progressively diverse $[25,26,33,35$, 36]. Fecal microbiome analyses of children and adults of the Amazonas of Venezuela, rural Malawi, and US metropolitan areas indicated that the phylogenic composition and the functional maturation of bacterial communities shift towards adult-like configuration during the first 3 years of childhood [25]. After that age, the gut appears to be protected from microbial colonization, including from supplementation with a cocktail of 11 strains of Bifidobacterium and Lactobacillus [37], in contrast to having lifelong effects from the diet $[34,38]$.

The gut microbiota of adult humans is dominated by the Firmicutes and Bacteroidetes phyla and smaller proportions of Actinobacteria, Proteobacteria, and Verrucomicrobia [39]. Sequencing analyses of human fecal metagenomes from four countries identified well-defined and robust microbial communities (termed enterotypes) represented by different levels of three genera: Bacteroides, Prevotella and Ruminococcus [40]. The three enterotypes do not correlate with gender, nationality, body mass index or age. However, the existence of these robust enterotypes remains debatable and instead the diet is considered to be a major driver of microbial composition and function [38, 41] (also see commentary by Jeffery et al., [42]). Moreover, the enterotypes have not been identified in a large study of healthy and frail elderly people [13]. Recently, the study of Italian centenarians (99-104 old), and semi-supercentenarian (105-109 old) suggested the presence of a different core microbiota dominated with Ruminococcaceae, Lachnospiraceae and Bacteridaceae families, which decrease with aging [19]. An independent study of Chinese centenarians revealed a negative association between extreme aging and the abundance of Coprococcus, Roseburia and Faecalibacterium genera, belonging to the Lachnospiraceae and Ruminococcaceae families [43, 44]. Despite differences in race, lifestyle and diet of the centenarians, the two studies found 11 shared features among the top 50 microbes, such as comparable changes in members of Blautia, Clostridium cluster XIVa, Faecalibacterium, Escherichia_Shigella, unclassified Lachnospiraceae, Ruminococcaceae, and Erysipelotrichaceae. Other groups reported that the enrichment of Bacteroidetes and Protobacteria abundances and decrease in species of Bifidobacteria and Lactobacilli in aged people [18, 45-50]. Interestingly, the Italian and Chinese study of centenarians found longevity increases microbial community richness (Chao index and 
observed operational taxonomic units, OTUs) and the abundance of subdominant but health-related bacterial genera and families, such as Oscillospira, Christensenellaceae, Akkermansia and Bifidobacterium [19, 44]. This presumably implies a healthy state of the elderly cohorts because Oscillospira and Christensenellaceae control leanness and decrease certain inflammatory diseases in humans [51, 52]. A. muciniphila protects the intestinal epithelial integrity, supports beneficial SCFA-producing bacteria and reduces inflammation and metabolic impairments such as insulin resistance [53, 54]. Bifidobacterium generates lactate and SCFA acid acetate and reduces pro-inflammatory microbes [55]. The aging-associated increase of Oscillospira, when compared to children and middle-aged adults, was also noted in recent multivariant unsupervised reanalysis of $16 \mathrm{~S}$ DNA sequencing data from 371 people ranging from newborn babies to centenarians [56].

Overall, despite significant interindividual variability and influence of external factors such as diet, medications, type of exercise or mobility, and the geographical locations of the host $[13,25]$, the composition of the gut microbiota changes progressively as people age [56]. Although the health status of subjects is often not reported, we can conclude that unlike young and middle-aged adults, the gut of elderly people is reduced in beneficial and enriched in pro-inflammatory commensal microbes. This change is presumably "intrinsic" to the aging process, as it also occurs in genetically homogeneous mice aged and fed in the same condition. Aged mice exhibit a decrease in beneficial gut bacteria, such as A. muciniphila and SCFA-producers in Clostridium members of cluster IV, and an increase pro-inflammatory microbes [53]. The changes in the gut microbiota composition with age are illustrated in Fig. 1.

\section{Does the alteration of the gut microbiota affect the health of elderly people?}

Metchnikoff's original concept published over a century ago explains the disabilities of elderly people by accumulation of "putrefactive bacterial autotoxins", which leaked from their colon. He postulated that this leakage results in the conversion of phagocytes into destroyers of healthy tissues [2]. This idea was confirmed centuries later by demonstrating the importance of chronic inflammation on activation as well dysfunction of phagocytic cells. Fecal microbiota markedly differs in people living in community-dwelling and long-term care nursing facilities [12, 13, 57, 58], consistent with its role in healthy aging. Dysbiosis, increased microbiota instability and loss of its members in community-dwelling adults can diminish numerous benefits of commensal microbes to the host [59]. This probably explains why decrease of the gut microbial diversity escalates as the frailty of elderly humans increases $[18,60-62]$, positively associating the gut dysbiosis of community-dwelling adults and the biological, but not chronological, age [60, 62]. Fecal microbiota of elderly frail people and aged mice is enriched in the Bacteroidetes phylum and the Oscillibacter and Alistipes genera and Eubacteriaceae family and is reduced in Faecalibacterium and Lactobacillus [12-14, 18]. Similarly, our group reported that elderly macaques and mice acquire increased insulin resistance because of gut dysbiosis, such as marked decrease of beneficial commensals and their metabolite SCFAs in the gut and in the circulation and increase of pro-inflammatory microbes [53]. A primary driver of this process in aged mice was Akkermansia, whose decrease caused intestinal leakage and activation of CCR2 $2^{+}$monocytes to convert innate $\mathrm{B} 1 \mathrm{a} \mathrm{B}$ cells into pathogenic $4-1 \mathrm{BBL}^{+} \mathrm{TNF}^{+} \mathrm{B}$ cells (termed 4BL cells), which in turn induced insulin resistance via the 4-1BBL/4-1BB axis [53]. Conversely and despite lifestyle and racial differences, both Chinese and Italian centenarians show an increase of the alpha diversity in the top 500 OUTs $[19,44]$ (although the Simpson reciprocal index of diversity was reduced in Italian centenarians [63]), suggesting that healthy aging may benefit from the retention of richness of commensal microbes in the gut. Overall, the decreased diversity of the intestinal commensal microbes, which often manifests in reduction of beneficial and enrichment of pro-inflammatory members, can have a detrimental consequence in healthy aging and thus longevity.

\section{The importance of microbial metabolites such as short chain fatty acids in immunity}

Although the composition of the gut microbiota changes with age, the core of microbiota does not age per se [18]. Fecal DNA sequencing and PCR analyses mostly reveal change in abundance of its individual members, which as a part of a symbiotic ecosystem then affects the microbial community and their crosstalk with the host. The abundance of Bifidobacterium-dominated commensals in the gut microbiota of breast-fed infants, which generates lactate and a beneficial SCFA (acetate) from milk oligosaccharides, progressively decreases together with the diversification of the microbial community after weaning and transition to adulthood [25]. Their decrease in young adults is complemented by other bacteria that metabolizes fibers to SCFAs, such as by their key producer Firmicutes [64, 65]. However, both Bifidobacterium and some members of Firmicutes, such as Clostridium clusters IV (Ruminococcus obeum et rel., Roseburia intestinalis et rel., E. ventriosum et rel., E. rectale et rel., E. hallii et rel.) and of Clostridium cluster XIVa, (Papillibacter cinnamovorans et rel., and F. prausnitzii et rel.), are decreased in aging and in centenarians [63]. This explains why the abundance of Bifidobacterium inversely 
correlates with inflammaging in elderly people and centenarians $[63,66]$, consistent with their benefit in prolonging longevity of mice as inhibitors of proinflammatory cytokines (for example from macrophages) and colonic senescence, and inducers of colonic tight junctions and mucus production [55, 67].

SCFAs provide energy to commensal microbes, immune cells and the colonic epithelium, and induce production of mucus e.g., they protect the epithelial barrier functions, support the growth of various beneficial commensal microbes, and promote immune tolerance and gut homeostasis [68]. SCFAs also regulate immune responses by directly stimulating immune cells. In airways of patients with cystic fibrosis, the increase of SCFAs, such as acetate, propionate, and butyrate, associates positively with neutrophil infiltration in the sputum and negatively with expansion of Pseudomonas aeruginosa. The SCFAs induce production of IL-8/CXCL8 and release of granulocyte-macrophage colony-stimulating factor (GM-CSF) and granulocyte colony-stimulating factor (G-CSF), which are needed in the recruitment and persistence of neutrophils, while inhibiting the synthesis of nitric oxide synthase involved in airway inflammation [69]. However, the increase of SCFAs, which can be achieved by feeding with a high-fiber diet, inhibits neutrophil recruitment and consequently airway inflammation and thereby enhances survival in influenza-infected mice [22]. In this study, SCFAs also impair infiltration of neutrophils in the lung airways because they recruit bone marrow-derived $\mathrm{Ly}^{-} \mathrm{c}^{-}$patrolling monocytes, which upon their differentiation into alternatively activated macrophages reduce expression of neutrophilrecruiting CXCL1 chemokine as well activate effector functions of $\mathrm{CD}^{+} \mathrm{T}$ cells. Extracellular SCFAs are also utilized as substrates for $\beta$-oxydation of fatty acids in $\mathrm{CD}^{+} \mathrm{T}$ cell [70] and colonocyte metabolism [71] after they are taken up by several G-protein coupled receptors (GPRs). Butyrate uncouples the tricarboxylic acid cycle from glycolytic input and enhances memory potential and recall responses of antigen-primed $\mathrm{CD}^{+} \mathrm{T}$ cells via GPR41, and GPR43 [70]. By targeting GPR109A [72], GPR41 and GPR43 [73-75], which are expressed on the surface of macrophages, dendritic cells and neutrophils, butyrate induces anti-inflammatory responses [76]. Similarly, through the interaction with GPR109A, it promotes anti-inflammatory pathways in colonic macrophages and dendritic cells and induces differentiation of Tregs and IL10-producing $\mathrm{T}$ cells [77]. Butyrate upregulates expression of transcription factor Blimp-1 and IL-10 production in Th1 $\mathrm{CD}^{+} \mathrm{T}$ cells (without affecting conversion of Tregs or Th17 cells) via GPR43 and controls colitis [78]. SCFAs from Clostridium species clusters IV and XIV induce and regulate colonic FoxP3 ${ }^{+}$Tregs $[79,80]$, although other SCFA non-producer microbes also support this process as they are required in the restoration of Tregs in GF mice after butyrate supplementation [9]. Butyrate is a potent inhibitor of histone deacetylases (HDACs), and its internalization via the above-noted GPRs or other unknown pathways causes epigenetic modifications of nonhematopoietic and hematopoietic cells [81-85], promoting anti-inflammatory immune responses through inhibition of the production of multiple pro-inflammatory cytokines (IFN- $\gamma$, IL-6, IL1- $\beta$ ) and induction of the release of antiinflammatory IL-10 and TGF- $\beta$ [86]. As an inhibitor of HDAC3, butyrate induces differentiation of monocytes to macrophages as well as metabolic and transcriptional changes in macrophages enhancing their bactericidal functions [87]. SCFAs inactivate nuclear factor- $\mathrm{kB}(\mathrm{NF}-\mathrm{kB})$ and reduce production of TNF $\alpha$ in peripheral blood mononuclear cells and neutrophils [88, 89]. By supporting differentiation of antibody-producing B cells, SCFAs increase both intestinal IgA and systemic IgG responses to combat pathogens [90]. Upon entering the bloodstream and reaching distant organ sites such as brain and lungs, SCFAs control systemic immune responses [91]. By regulating Tregs in the lungs, SCFAs protect against allergic airway diseases $[21,92,93]$. In the brain, SCFAs directly affect microglia maturation and function [94] and thereby reduce neuroinflammation [95]. However, SCFAs can also exert pro-inflammatory responses under certain conditions $[69,96,97]$. For instance, SCFAs can aggravate colitis-associated inflammation in mice by inducing expression of T-bet and IFN $\gamma$ in Tregs and in conventional $\mathrm{CD}^{+}{ }^{+} \mathrm{T}$ cells [96]. Also, SCFAs can exacerbate inflammation in airway epithelial cells [69], and the generation of IL-17 and IFN $\gamma$ producing $\mathrm{T}$ cells in the CNS in mice with multiple sclerosis [97]. In the mouse model of Parkinson's disease (PD), SCFAs are linked to acceleration of pathogenic $\alpha$-synuclein $(\alpha \operatorname{Syn})$ aggregation and motor deficits [98]. The different functions of SCFAs in the gut microbiota are illusrated in Fig. 2.

\section{Alteration of the SCFA levels in elderly people}

As discussed above, SCFAs are produced by a number of bacterial populations working in concert with a community of commensals [99]. For example, A. muciniphila not only supports growth of other commensal microbes by liberating oligosaccharides as well maintaining the mucus layer, but also induces butyrate production from Anaerostipes caccae, Eubacterium hallii, and Faecalibacterium prausnitzii, while $E$. hallii upregulates pseudovitamin B12 to stimulate propionate production from $A$. muciniphila [100]. Butyrate improves barrier function of the intestinal epithelial cells (IECs) and protects them from $C$. difficile toxin damage by activating hypoxiainducible factor $1 \alpha$ [101]. This probably explains why the gut of centenarians retains high levels of butyrate and has increased quantities of the butyrate producers 
Anaerotruncus colihominis et rel. (from Clostridium cluster IV) and Eubacterium limosum et rel. (from Clostridium cluster XV), Bifidobacterium and other the health-associated bacteria, such as Akkermansia and Christensenellaceae [19, 44, 99]. A. muciniphila and its outer membrane protein Amuc_1100* also stimulate IECs to induce mucus production necessary for protection of the intestinal barrier integrity and the support of other beneficial commensals [102-104]. Therefore, the decrease of this important bacterium causes gut dysbiosis and impairs the intestinal epithelial integrity, which increases gut leakiness and systemic endotoxemia. A resulting chronic proinflammatory state with higher levels of circulating IL-6, IFNs, TNF $\alpha$, and IL-1 then promotes poor fitness and frailty in elderly humans $[16,17,44,63,105]$. Our group recently reported that the decrease of A. muciniphila in the gut of aged mice triggers a chain of inflammatory events that manifests in the increase of insulin resistance [53]. It starts from the reduction of the colonic mucin layer, which presumably leads to the loss of commensal bacteria producing butyrate, such as I. butyriciproducens, F. prausnitzii, R. faecis, and A. butyraticus, and thus a reduction in SCFAs, particularly butyrate, in the gut lumen and in the circulation. The decrease of this important source of energy to colonocytes and suppressor of proinflammatory and pathogenic commensal bacteria [106] further enhances dysbiosis and gut leakage in aged mice, thereby sustaining inflammaging [53]. Because butyrate is also a potent inhibitor of TLR4 signaling [107], its decrease in the circulation enables bacterial stimuli, such as endotoxin, to freely induce inflammation in the omentum and to upregulate expression of 4-1BB, CD40L, and IFN- $\gamma$ in BM-derived inflammatory $\mathrm{CCR}^{+}$monocytes. Upon infiltration into the omentum, these monocytes convert innate B1a B cells into 4BL cells [53] via 4-1BBL, CD40, and IFN $\gamma \mathrm{R} 1$ signaling. The 4BL cells then promote insulin resistance in aged hosts, at least in part, utilizing the $4-1 \mathrm{BBL} / 4-1 \mathrm{BB}$ axis [53]. A similar pathway appears to exist in elderly humans and macaques, as their increased insulin resistance associates with $4 \mathrm{BL}$ cells induced by aging-activated monocytes $[53,108,109]$. In elderly people, the level of SCFAs from carbohydrate fermentation is decreased while metabolites from protein fermentation (branched fatty acids, ammonia and phenols) are increased, indicating a shift from saccharolytic fermentation to unfavorable proteolytic activities $[110,111]$. This shift occurs progressively as elderly people age [99], and can be accelerated upon the use of antibiotics or with low-fiber diets [110, 112, 113]. The decrease in the level of SCFAs enhances susceptibility to opportunistic bacterial species and pathogens. For instance, treatment with the antibiotic cefoperazone reduces SCFAs and thereby increases colonization of the fungus Candida albicans in mice [114]. Similarly, antibiotic-induced depletion of butyrate-producing microbes decreases butyrate levels, which leads to epithelial oxygenation and expansion of aerobic bacteria such as Escherichia coli and Salmonella enterica [106, 115]. Mechanistically, the colonocyte oxygenation is primarily linked to decreased activity of the intracellular butyrate sensor peroxisome proliferatoractivated receptor $\gamma$ (PPAR- $\gamma$ ), which leads to activation of iNOS and increase of nitrate levels [106]. The mechamisms in which gut dysbiosis increases age-related diseases are illustrated in Fig. 3.

\section{Contribution of other microbial factors in inflammaging}

The gut microbiota controls local immune responses, such as the development and regulation of the intestinal immune responses [116]. Germ-free rodents show an alteration in the development of the gut-associated lymphoid tissues (GALT) and maturation of isolated lymphoid follicles (ILFs), leading to significantly lower numbers of T cells, including Tregs, and IgA-producing B cells [116, 117]. On the other hand, dysbiosis induces systemic inflammation, explaining relatively high plasma levels of MCP-1/CCL2 [118], IL-8/CXCL8, TNF $\alpha$, IL-6, and Creactive protein (CRP) in elderly people living in longterm care facilities [13]. Underscoring the importance of gut microbiota, the inflammatory phenotype in aging is also transferable, as GF mice show signs of inflammaging after transplantation with fecal microbiota of from aged, but not young, mice [119]. Similarly, the fecal transplantation from aged, but not young, mice induces the genration of $4 \mathrm{BL}$ cells in GF mice, primarily due to absence of A. muciniphila and SCFA-producer bacteria [53]. However, gut microbiota modulates inflammaging through a plethora of additional mechanisms. For instance, the gut of long lived elderly people is decreased in proinflammatory $F$. prausnitzii and is increased about 15-fold in E. limosum [63]. Elderly people and centenarians also markedly decrease abundance of Bifidobacterium genus in the gut $[25,63,66]$, presumably explaining increased inflammaging and consequent aging-associated morbidity and mortality. Bifidobacterium is an important producer of lactate, which affects acidity of the environment, and polyamines that scavenge reactive oxygen species (ROS). It also induces stress-response genes, regulates NFkB activation, inhibits pro-inflammatory cytokines from macrophages, suppresses colonic senescence, maintains colonic tight junctions and mucus production, and even prolongs longevity in mice [55, 67], implying that Bifidobacterium supplementation may also reverse agingassociated impairments in elderly humans. However, recent systematic reviews and meta-analyses of randomized numerous probiotic trials revealed no significant benefit in protection from infection, improving NK cell function, quality of life and mortality of elderly people as compared 
with placebo controls [120, 121]. Despite importance of probiotics, the key question remains unresolved - whether they can efficiently colonize the gut of adults and elderly people. Recent study failed to find the colonization evidence in adults supplemented with a cocktail of 11 Bifidobacterium and Lactobacillus strains failed [37]. It is also unclear whether orally given probiotics can survive acidity of the stomach and competition from the commensal microbiota. Lastly, if the bacterial decrease in aging is caused by the immune system, attempts to restore them, for example by supplementing Bifidobacterium, may instead accelerate their loss due to activation of the immune response.

\section{Concluding remarks}

Despite significant interindividual and lifestyle differences, the composition of the gut microbiota of elderly humans markedly differs from that of young and middle-aged adults. The compositional shift coincides with the onset of immune dysregulation and manifestation of aging-associated pathologies, e.g. $\geq 70$ years of age. In elderly, particularly frail people, the composition of the gut microbiota shows signs of dysbiosis, such as a marked decrease in diversity of its population due to the accumulation of proinflammatory commensals and reduction of beneficial microbes. The decrease of beneficial microbes, particularly supporters of mucin production and producers of SCFAs, appears to be essential in triggering a chain of inflammatory events, such as the impairment of intestinal barrier integrity and increase of gut leakiness, endotoxemia and subsequent inflammaging and agingassociated morbidities. As in aged fruit flies where intestinal permeability increases mortality [122, 123], we therefore propose that the gut dysbiosis and leakiness is a major cause of premature death in elderly people. Consistent with ancient philosophers and Elie Metchnikoffs vision and as lifestyle improvements show, extrinsic manipulations can control the ills of the elderly to maintain healthy aging. This concept is validated in modeling studies in rodents, revealing that aging-associated pathologies are reversible. For example, the gut microbiota-induced inflammaging and consequent increased insulin resistance is reversed by restoring $A$. muciniphila, supplementing with butyrate, or inactivating 4BL cells and monocytes in aged mice and macaques [53]. On the other hand, little is known about the cause $(-s)$ of dysbiosis, the safety and potential health risk of microbiota-based interventions in elderly people. Further research is needed to fully understand the benefit of probiotics and their use in humans.

\section{Acknowledgments}

We are grateful to Mrs. A. Lustig (NIA) for helpful comments and proofreading.
Conflict of interest

The authors do not have any conflict of interest.

Authors' contributions

E.M. and A.B. analyzed literature and wrote the paper; A.B. conceived and supervised study. Both authors read and approved the final manuscript.

\section{Funding}

This research was supported by the Intramural Research Program of the National Institute on Aging, $\mathrm{NIH}$.

\section{Availability of data and materials}

Yes

\section{Ethics approval and consent to participate} N/A

\section{Consent for publication}

Yes

\section{Competing interests}

None.

Received: 8 October 2020 Accepted: 21 December 2020 Published online: 05 January 2021

\section{References}

1. Galen, et al. Hygiene, Loeb classical library, vol. 2. Cambridge: Harvard University Press; 2018.

2. Metchnikoff E, Mitchell PC. The prolongation of life; optimistic studies. London: W. Heinemann; G.P. Putnam's Sons. xx; 1907. p. 343.

3. Gill SR, et al. Metagenomic analysis of the human distal gut microbiome. Science. 2006;312(5778):1355-9.

4. Honda K, Littman DR. The microbiota in adaptive immune homeostasis and disease. Nature. 2016;535(7610):75-84

5. Atarashi $\mathrm{K}$, et al. Th17 cell induction by adhesion of microbes to intestinal epithelial cells. Cell. 2015;163(2):367-80.

6. Atarashi $\mathrm{K}$, et al. Treg induction by a rationally selected mixture of clostridia strains from the human microbiota. Nature. 2013;500(7461):232-6.

7. Satoh-Takayama N, et al. Bacteria-induced group 2 innate lymphoid cells in the stomach provide immune protection through induction of $\operatorname{lgA}$. Immunity. 2020;52(4):635-649 e4.

8. Hansson J, et al. Influence of gut microbiota on mouse B2 B cell ontogeny and function. Mol Immunol. 2011;48(9-10):1091-101.

9. Furusawa $Y$, et al. Commensal microbe-derived butyrate induces the differentiation of colonic regulatory T cells. Nature. 2013;504(7480):446-50.

10. Tanoue T, et al. A defined commensal consortium elicits CD8 T cells and anti-cancer immunity. Nature. 2019;565(7741):600-5.

11. Magrone T, Jirillo E. The interaction between gut microbiota and agerelated changes in immune function and inflammation. Immun Ageing. 2013;10(1):31.

12. van Tongeren SP, et al. Fecal microbiota composition and frailty. Appl Environ Microbiol. 2005;71(10):6438-42.

13. Claesson MJ, et al. Gut microbiota composition correlates with diet and health in the elderly. Nature. 2012;488(7410):178-84.

14. Langille MG, et al. Microbial shifts in the aging mouse gut. Microbiome. 2014;2(1):50.

15. Jeffery IB, Lynch DB, O'Toole PW. Composition and temporal stability of the gut microbiota in older persons. ISME J. 2016;10(1):170-82.

16. Hearps $A C$, et al. Aging is associated with chronic innate immune activation and dysregulation of monocyte phenotype and function. Aging Cell. 2012; 11(5):867-75.

17. Bouchlaka $\mathrm{MN}$, et al. Aging predisposes to acute inflammatory induced pathology after tumor immunotherapy. J Exp Med. 2013;210(11):2223-37.

18. O'Toole PW, Jeffery IB. Gut microbiota and aging. Science. 2015;350(6265): 1214-5.

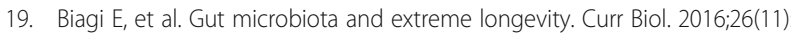
$1480-5$.

20. Brestoff JR, Artis D. Commensal bacteria at the interface of host metabolism and the immune system. Nat Immunol. 2013;14(7):676-84. 
21. Trompette A, et al. Gut microbiota metabolism of dietary fiber influences allergic airway disease and hematopoiesis. Nat Med. 2014;20(2):159-66.

22. Trompette A, et al. Dietary fiber confers protection against flu by shaping Ly6c(-) patrolling monocyte hematopoiesis and CD8(+) T cell metabolism. Immunity. 2018;48(5):992-1005 e8.

23. Arpaia N, et al. Metabolites produced by commensal bacteria promote peripheral regulatory T-cell generation. Nature. 2013;504(7480):451-5.

24. Ivanov II, et al. Induction of intestinal Th17 cells by segmented filamentous bacteria. Cell. 2009;139(3):485-98.

25. Yatsunenko T, et al. Human gut microbiome viewed across age and geography. Nature. 2012;486(7402):222-7.

26. Backhed F, et al. Dynamics and stabilization of the human gut microbiome during the first year of life. Cell Host Microbe. 2015;17(6):852.

27. Bokulich NA, et al. Antibiotics, birth mode, and diet shape microbiome maturation during early life. Sci Transl Med. 2016;8(343):343ra82.

28. Dominguez-Bello MG, et al. Delivery mode shapes the acquisition and structure of the initial microbiota across multiple body habitats in newborns. Proc Natl Acad Sci U S A. 2010;107(26):11971-5.

29. Hill CJ, et al. Evolution of gut microbiota composition from birth to 24 weeks in the INFANTMET cohort. Microbiome. 2017;5(1):4.

30. Nagpal R, et al. Evolution of gut Bifidobacterium population in healthy Japanese infants over the first three years of life: a quantitative assessment. Sci Rep. 2017;7(1):10097.

31. Nagpal R, et al. Gut dysbiosis following C-section instigates higher colonisation of toxigenic Clostridium perfringens in infants. Benefic Microbes. 2017;8(3):353-65.

32. Penders $\mathrm{J}$, et al. Factors influencing the composition of the intestinal microbiota in early infancy. Pediatrics. 2006;118(2):511-21.

33. Tsuji $\mathrm{H}$, et al. Molecular monitoring of the development of intestinal microbiota in Japanese infants. Benefic Microbes. 2012;3(2):113-25.

34. De Filippo C, et al. Impact of diet in shaping gut microbiota revealed by a comparative study in children from Europe and rural Africa. Proc Natl Acad Sci U S A. 2010;107(33):14691-6.

35. Favier CF, et al. Molecular monitoring of succession of bacterial communities in human neonates. Appl Environ Microbiol. 2002;68(1): 219-26.

36. Cheng J, et al. Discordant temporal development of bacterial phyla and the emergence of core in the fecal microbiota of young children. ISME J. 2016; 10(4):1002-14.

37. Zmora $\mathrm{N}$, et al. Personalized gut mucosal colonization resistance to empiric probiotics is associated with unique host and microbiome features. Cell. 2018;174(6):1388-1405 e21.

38. David LA, et al. Diet rapidly and reproducibly alters the human gut microbiome. Nature. 2014:505(7484):559-63.

39. Eckburg PB, et al. Diversity of the human intestinal microbial flora. Science. 2005;308(5728):1635-8.

40. Arumugam $\mathrm{M}$, et al. Enterotypes of the human gut microbiome. Nature 2011:473(7346):174-80

41. Muegge $\mathrm{BD}$, et al. Diet drives convergence in gut microbiome functions across mammalian phylogeny and within humans. Science. 2011;332(6032): 970-4.

42. Jeffery $I B$, et al. The microbiota link to irritable bowel syndrome: an emerging story. Gut Microbes. 2012;3(6):572-6.

43. Wang $\mathrm{N}$, et al. Enriched taxa were found among the gut microbiota of centenarians in East China. PLoS One. 2019:14(10):e0222763.

44. Kong F, et al. Gut microbiota signatures of longevity. Curr Biol. 2016;26(18): R832-3.

45. He F, et al. Differences in composition and mucosal adhesion of bifidobacteria isolated from healthy adults and healthy seniors. Curr Microbiol. 2001;43(5):351-4

46. Hopkins MJ, Macfarlane GT. Changes in predominant bacterial populations in human faeces with age and with Clostridium difficile infection. J Med Microbiol. 2002;51(5):448-54.

47. Makivuokko $\mathrm{H}$, et al. The effect of age and non-steroidal anti-inflammatory drugs on human intestinal microbiota composition. Br J Nutr. 2010;103(2): 227-34

48. Mitchell EL, et al. Reduced intestinal motility, mucosal barrier function, and inflammation in aged monkeys. J Nutr Health Aging. 2017;21(4):354-61.

49. Odamaki $\mathrm{T}$, et al. Age-related changes in gut microbiota composition from newborn to centenarian: a cross-sectional study. BMC Microbiol. 2016;16:90.
50. Rea MC, et al. Clostridium difficile carriage in elderly subjects and associated changes in the intestinal microbiota. J Clin Microbiol. 2012; 50(3):867-75.

51. Konikoff T, Gophna U. Oscillospira: a central, enigmatic component of the human gut microbiota. Trends Microbiol. 2016;24(7):523-4.

52. Waters JL, Ley RE. The human gut bacteria Christensenellaceae are widespread, heritable, and associated with health. BMC Biol. 2019;17(1):83.

53. Bodogai $\mathrm{M}$, et al. Commensal bacteria contribute to insulin resistance in aging by activating innate B1a cells. Sci Transl Med. 2018;10(467).

54. Schneeberger $M$, et al. Akkermansia muciniphila inversely correlates with the onset of inflammation, altered adipose tissue metabolism and metabolic disorders during obesity in mice. Sci Rep. 2015;5:16643.

55. Sela DA, et al. The genome sequence of Bifidobacterium longum subsp. infantis reveals adaptations for milk utilization within the infant microbiome. Proc Natl Acad Sci U S A. 2008;105(48):18964-9.

56. Xu C, Zhu H, Qiu P. Aging progression of human gut microbiota. BMC Microbiol. 2019;19(1):236

57. Kinross J, Nicholson JK. Gut microbiota: dietary and social modulation of gut microbiota in the elderly. Nat Rev Gastroenterol Hepatol. 2012; 9(10):563-4.

58. Collino $\mathrm{S}$, et al. Metabolic signatures of extreme longevity in northern Italian centenarians reveal a complex remodeling of lipids, amino acids, and gut microbiota metabolism. PLoS One. 2013;8(3):e56564.

59. Rooks MG, Garrett WS. Gut microbiota, metabolites and host immunity. Nat Rev Immunol. 2016;16(6):341-52.

60. Maffei VJ, et al. Biological aging and the human gut microbiota. J Gerontol A Biol Sci Med Sci. 2017;72(11):1474-82

61. Kim S, Jazwinski SM. The gut microbiota and healthy aging: a mini-review. Gerontology. 2018;64(6):513-20.

62. Jackson MA, et al. Signatures of early frailty in the gut microbiota. Genome Med. 2016;8(1):8.

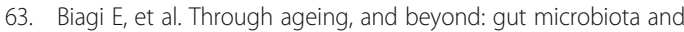
inflammatory status in seniors and centenarians. PLoS One. 2010;5(5): e10667.

64. Barcenilla A, et al. Phylogenetic relationships of butyrate-producing bacteria from the human gut. Appl Environ Microbiol. 2000;66(4):1654-61.

65. Louis P, Flint HJ. Diversity, metabolism and microbial ecology of butyrateproducing bacteria from the human large intestine. FEMS Microbiol Lett. 2009;294(1):1-8.

66. Mueller $\mathrm{S}$, et al. Differences in fecal microbiota in different European study populations in relation to age, gender, and country: a cross-sectional study. Appl Environ Microbiol. 2006;72(2):1027-33.

67. Matsumoto $\mathrm{M}$, et al. Longevity in mice is promoted by probiotic-induced suppression of colonic senescence dependent on upregulation of gut bacterial polyamine production. PLoS One. 2011;6(8):e23652.

68. Correa-Oliveira $R$, et al. Regulation of immune cell function by short-chain fatty acids. Clin Transl Immunol. 2016;5(4):e73.

69. Ghorbani $P$, et al. Short-chain fatty acids affect cystic fibrosis airway inflammation and bacterial growth. Eur Respir J. 2015;46(4):1033-45.

70. Bachem A, et al. Microbiota-derived short-chain fatty acids promote the memory potential of antigen-activated CD8(+) T cells. Immunity. 2019;51(2): 285-297 e5.

71. Donohoe $\mathrm{DR}$, et al. The microbiome and butyrate regulate energy metabolism and autophagy in the mammalian colon. Cell Metab. 2011; 13(5):517-26.

72. Thangaraju M, et al. GPR109A is a G-protein-coupled receptor for the bacterial fermentation product butyrate and functions as a tumor suppressor in colon. Cancer Res. 2009;69(7):2826-32.

73. Brown AJ, et al. The orphan G protein-coupled receptors GPR41 and GPR43 are activated by propionate and other short chain carboxylic acids. J Biol Chem. 2003:278(13):11312-9.

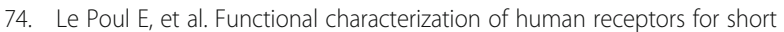
chain fatty acids and their role in polymorphonuclear cell activation. J Biol Chem. 2003;278(28):25481-9.

75. Ulven T. Short-chain free fatty acid receptors FFA2/GPR43 and FFA3/ GPR41 as new potential therapeutic targets. Front Endocrinol (Lausanne). 2012;3:111.

76. Goncalves P, Araujo JR, Di Santo JP. A cross-talk between microbiotaderived short-chain fatty acids and the host mucosal immune system regulates intestinal homeostasis and inflammatory bowel disease. Inflamm Bowel Dis. 2018;24(3):558-72 
77. Singh N, et al. Activation of Gpr109a, receptor for niacin and the commensal metabolite butyrate, suppresses colonic inflammation and carcinogenesis. Immunity. 2014;40(1):128-39.

78. Sun M, et al. Microbiota-derived short-chain fatty acids promote Th1 cell IL-10 production to maintain intestinal homeostasis. Nat Commun. 2018;9(1):3555.

79. Atarashi $\mathrm{K}$, et al. Induction of colonic regulatory $T$ cells by indigenous Clostridium species. Science. 2011;331(6015):337-41.

80. Smith PM, et al. The microbial metabolites, short-chain fatty acids, regulate colonic Treg cell homeostasis. Science. 2013;341(6145):569-73.

81. Daroqui MC, Augenlicht LH. Transcriptional attenuation in colon carcinoma cells in response to butyrate. Cancer Prev Res (Phila). 2010;3(10):1292-302.

82. Davie JR. Inhibition of histone deacetylase activity by butyrate. J Nutr. 2003; 133(7 Suppl):2485S-93S.

83. Sekhavat A, Sun JM, Davie JR. Competitive inhibition of histone deacetylase activity by trichostatin A and butyrate. Biochem Cell Biol. 2007;85(6):751-8.

84. Thangaraju $\mathrm{M}$, et al. Colon cancer cells maintain low levels of pyruvate to avoid cell death caused by inhibition of HDAC1/HDAC3. Biochem J. 2009; 417(1):379-89.

85. Wong JM, et al. Colonic health: fermentation and short chain fatty acids. J Clin Gastroenterol. 2006;40(3):235-43.

86. Mowat AM, Agace WW. Regional specialization within the intestinal immune system. Nat Rev Immunol. 2014;14(10):667-85.

87. Schulthess J, et al. The short chain fatty acid butyrate imprints an antimicrobial program in macrophages. Immunity. 2019;50(2):432-445 e7.

88. Usami M, et al. Butyrate and trichostatin A attenuate nuclear factor kappaB activation and tumor necrosis factor alpha secretion and increase prostaglandin E2 secretion in human peripheral blood mononuclear cells. Nutr Res. 2008;28(5):321-8.

89. Vinolo MA, et al. Suppressive effect of short-chain fatty acids on production of proinflammatory mediators by neutrophils. J Nutr Biochem. 2011;22(9): 849-55.

90. Kim M, et al. Gut microbial metabolites fuel host antibody responses. Cell Host Microbe. 2016;20(2):202-14.

91. Beura LK, et al. Normalizing the environment recapitulates adult human immune traits in laboratory mice. Nature. 2016;532(7600):512-6.

92. Thorburn AN, et al. Evidence that asthma is a developmental origin disease influenced by maternal diet and bacterial metabolites. Nat Commun. 2015:6:7320.

93. Zhang $Z$, et al. Dietary fiber intake regulates intestinal microflora and inhibits ovalbumin-induced allergic airway inflammation in a mouse model. PLoS One. 2016;11(2):e0147778.

94. Erny D, et al. Host microbiota constantly control maturation and function of microglia in the CNS. Nat Neurosci. 2015;18(7):965-77.

95. Sherry $\mathrm{CL}$, et al. Sickness behavior induced by endotoxin can be mitigated by the dietary soluble fiber, pectin, through up-regulation of IL-4 and Th2 polarization. Brain Behav Immun. 2010;24(4):631-40.

96. Kespohl M, et al. The microbial metabolite butyrate induces expression of Th1-associated factors in CD4(+) T cells. Front Immunol. 2017:8:1036.

97. Park J, et al. Author correction: bidirectional regulatory potentials of shortchain fatty acids and their G-protein-coupled receptors in autoimmune neuroinflammation. Sci Rep. 2019;9(1):17511.

98. Sampson TR, et al. Gut microbiota regulate motor deficits and neuroinflammation in a model of Parkinson's disease. Cell. 2016;167(6): 1469-1480 e12.

99. den Besten $\mathrm{G}$, et al. The role of short-chain fatty acids in the interplay between diet, gut microbiota, and host energy metabolism. J Lipid Res. 2013;54(9):2325-40.

100. Belzer C, et al. Microbial metabolic networks at the mucus layer lead to diet-independent butyrate and vitamin B12 production by intestinal symbionts. mBio. 2017;8(5).

101. Fachi $J$, et al. Butyrate protects mice from Clostridium difficile-induced colitis through an HIF-1-dependent mechanism. Cell Rep. 2019;27(3):750761 e7.

102. Plovier $\mathrm{H}$, et al. A purified membrane protein from Akkermansia muciniphila or the pasteurized bacterium improves metabolism in obese and diabetic mice. Nat Med. 2017;23(1):107-13.

103. Derrien M, Belzer C, de Vos WM. Akkermansia muciniphila and its role in regulating host functions. Microb Pathog. 2017;106:171-81.

104. Derrien M, et al. The Mucin degrader Akkermansia muciniphila is an abundant resident of the human intestinal tract. Appl Environ Microbiol. 2008;74(5):1646-8.
105. Rampelli $S$, et al. Functional metagenomic profiling of intestinal microbiome in extreme ageing. Aging (Albany NY). 2013;5(12):902-12.

106. Byndloss MX, et al. Microbiota-activated PPAR-gamma signaling inhibits dysbiotic Enterobacteriaceae expansion. Science. 2017;357(6351):570-5.

107. Cox MA, et al. Short-chain fatty acids act as antiinflammatory mediators by regulating prostaglandin E (2) and cytokines. World J Gastroenterol. 2009; 15(44):5549-57.

108. Lee-Chang C, et al. Aging converts innate B1a cells into potent CD8+ T cell inducers. J Immunol. 2016;196(8):3385-97.

109. Lee-Chang $C$, et al. Accumulation of $4-1 B B L+B$ cells in the elderly induces the generation of granzyme- $B+C D 8+T$ cells with potential antitumor activity. Blood. 2014;124(9):1450-9.

110. Woodmansey EJ. Intestinal bacteria and ageing. J Appl Microbiol. 2007; 102(5):1178-86.

111. Salazar N, et al. Age-associated changes in gut microbiota and dietary components related with the immune system in adulthood and old age: a cross-sectional study. Nutrients. 2019;11(8).

112. Laurin $D$, et al. Fibre intake in elderly individuals with poor masticatory performance. J Can Dent Assoc. 1994;60(5):443-6, 449.

113. Tiihonen K, Ouwehand AC, Rautonen N. Human intestinal microbiota and healthy ageing. Ageing Res Rev. 2010;9(2):107-16.

114. Guinan J, et al. Antibiotic-induced decreases in the levels of microbialderived short-chain fatty acids correlate with increased gastrointestinal colonization of Candida albicans. Sci Rep. 2019;9(1):8872.

115. Rivera-Chavez F, et al. Depletion of butyrate-producing clostridia from the gut microbiota drives an aerobic luminal expansion of Salmonella. Cell Host Microbe. 2016;19(4):443-54.

116. Round JL, Mazmanian SK. The gut microbiota shapes intestinal immune responses during health and disease. Nat Rev Immunol. 2009;9(5):313-23.

117. Bouskra D, et al. Lymphoid tissue genesis induced by commensals through NOD1 regulates intestinal homeostasis. Nature. 2008;456(7221):507-10.

118. Conley MN, et al. Aging and serum MCP-1 are associated with gut microbiome composition in a murine model. PeerJ. 2016:4:e1854.

119. Fransen F, et al. Aged gut microbiota contributes to systemical inflammaging after transfer to germ-free mice. Front Immunol. 2017;8:1385.

120. Wachholz PA, et al. Effectiveness of probiotics on the occurrence of infections in older people: systematic review and meta-analysis. Age Ageing. 2018:47(4):527-36.

121. Gui $Q$, et al. Effects of probiotic supplementation on natural killer cell function in healthy elderly individuals: a meta-analysis of randomized controlled trials. Eur J Clin Nutr. 2020

122. Clark Rl, et al. Distinct shifts in microbiota composition during drosophila aging impair intestinal function and drive mortality. Cell Rep. 2015;12(10): 1656-67.

123. Rera M, Clark Rl, Walker DW. Intestinal barrier dysfunction links metabolic and inflammatory markers of aging to death in Drosophila. Proc Natl Acad Sci U S A. 2012;109(52):21528-33

\section{Publisher's Note}

Springer Nature remains neutral with regard to jurisdictional claims in published maps and institutional affiliations.

Ready to submit your research? Choose BMC and benefit from:

- fast, convenient online submission

- thorough peer review by experienced researchers in your field

- rapid publication on acceptance

- support for research data, including large and complex data types

- gold Open Access which fosters wider collaboration and increased citations

- maximum visibility for your research: over $100 \mathrm{M}$ website views per year

At BMC, research is always in progress.

Learn more biomedcentral.com/submissions 